\title{
Evidence-based Reconstruction of Kampo Medicine: Part I-Is Kampo CAM?
}

\author{
Katsutoshi Terasawa
}

Toyama Medical and Pharmaceutical University, Japan

\section{Introduction}

I would like to express my hearty congratulations at the launching of the new journal Evidence-based Complementary and Alternative Medicine (eCAM). I would also like to thank the Editors, Drs Tomio Tada, Edwin Cooper and Nobuo Yamaguchi, for inviting me to contribute a lecture series on the evidential basis of Kampo (Chinese herbal) medicine.

This series will run for several issues with the following main topics (in this issue the first three topics are mainly covered):

- Is Kampo CAM?

- The historical background of Kampo

- What characterizes Kampo?

- Herbal compositions of Kampo medicines

- Composition and analysis of Kampo medicines

- Sho (symptoms)

- Construction of evidence for Kampo, based on Sho

- Various designs for clinical trials to construct an evidential basis for Kampo

- Outline of the existing evidence of using Kampo for the treatment of various diseases

Preliminary notes for those who are not acquainted with Kampo: the term 'Kampo' is a Japanese name for Chinese herbal medicine. The difference between 'Chinese' Chinese herbal medicine and Kampo will be discussed in the historical overview section. Sho is broadly defined as Kampo diagnosis in the epistemic framework of Kampo's view of illness. The response to Kampo medicine is predicted by Sho.

For reprints and all correspondence: Professor Katsutoshi Terasawa, Department of Japanese-Oriental (Kampo) Medicine. Faculty of Medicine,Toyama Medical and Pharmaceutical University.

E-mail: terasawa@ms.toyama-mpu.ac.jp

Professor Katsutoshi Terasawa was born in Tokyo in 1944. He graduated in 1970 from the School of Medicine at Chiba University. He became Director of the Department of Japanese Oriental Medicine at Toyama Medical and Pharmaceutical University in 1979. He was Director of the School of Medicine (1999-2000) and then President of the Hospital and Vice-President (2002-2004) of Toyama Medical and Pharmaceutical University. He is now President of the Japan Society for Oriental Medicine and Director of the WHO Collaborating Center for Traditional Medicine.

\section{My Personal Path Towards the Integration of Two Medical Systems}

I thought it would be of interest, particularly for Western readers, to give my personal recollections on my scientific quest for the integration of medicines of the West and the East. It has been my life-long endeavor to search for ways to integrate these two medical systems with two completely different paradigms. The first prerequisite, of course, is to deeply understand both systems, or speak two different languages, as it were, like a bilingual who can speak English and Japanese with equal fluency. In Japan, the standard medical education firmly sticks to the system of Western medicine so that no medical doctor with 'bilingual' competence has been nurtured, at least institutionally. I am proud to say that I am one of the very few people who have overcome this difficulty. My motivation for aspiring to become a medical bilingual was two-fold: one was circumstantial and the other philosophical. From early childhood, I lived close to an uncle who ran a small private ophthalmology clinic. He was educated in Western medicine, but when he found no Western remedies for the eye diseases of many of his patients he did not hesitate to prescribe Kampo medicines. He was respected by many of his patients as a 'master' doctor. Watching my uncle made me feel that it was natural or practical to treat patients by any effective means, regardless of its origin in the East or West. My second motive was related to the Zeitgeist of the 1960s. Young people during the time of the Vietnam War wanted to call all aspects of the 'establishment' into question, whether political or cultural. Modern Western medicine was, and still is, the invincible establishment in medicine. At that time, I was a sort of dissident who did not like to see the remedies of which my uncle was a 'master' simply ignored as superstition by mainstream medicine. I thought that there must be a way through which the two kinds of medicine could be integrated. When I entered Chiba University in 1964, medical students had started a small private circle to study Oriental medicine. This gave me the opportunity to study the Kampo system in parallel with orthodox Western medical education.

I graduated from medical school in 1970, specializing in internal medicine. After three years in general internal medicine, I received further training in neurology. This study 
reminded me of the importance of the anatomy of the central nervous system, and I did research in this field for my $\mathrm{PhD}$ thesis, before returning to clinical practice in neurology.

While I underwent this thoroughly Western-style medical training, I struggled under severe time constrictions to maintain my ability in Kampo. I worked weekends as a part-time Kampo practitioner, trying to refine my 'bilingual' ability. Then, in 1979, a new clinical department was established in the new Toyama Medical and Pharmaceutical University Hospital, aiming to integrate Western and Kampo medicine. It was the first such department at a Japanese national university and I was invited to be the Director of this new small department. At that time, however, the atmosphere of the Japanese academic medical world was far from warm toward Kampo medicine. Many of my colleagues in internal medicine viewed me as something akin to a guru of a strange religious cult! Having failed to become a famous guru in supernatural healing, unfortunately, I have been engaged in the orthodox clinical practice, research and education in this field towards integration of Kampo with modern Western medicine for a quarter of a century.

I made it the basic policy of the clinical practice in our department to try Kampo-based remedies for patients whose problems were difficult to solve through conventional Western medicine. It is also our policy to evaluate the outcome using the conventional clinical examinations of Western medicine, including imaging technologies such as MRI. In any situation where the use of Western medicine is clearly indicated, like the exacerbation of systemic lupus erythematodes (SLE), we do not hesitate to resort to a conventional mode of therapy, such as pulse therapy by steroids.

On the research front, we have been trying to elucidate the cognition by Kampo of the pathological states that constitute the epidemiological foundation of Kampo medicine in Western scientific (or molecular biological) terms. We have also carried out various clinical trials, using methods such as double-blind control trials, to test the clinical efficacy of various Kampo prescriptions.

In our medical school, Kampo has been integrated into the curriculum and students are given an overview of Kampo through lectures and clinical practice alongside courses in Western medicine. Until now only our university has adopted this approach, but in 2003 the Ministry of Education and Science of Japan, the governmental department overseeing medical education, decided to recommend that all medical schools in Japan include a course giving an overview of Kampo within the core curriculum for medical students. This is truly an epoch-making decision in the history of modern Japanese medical education, which has completely ignored the traditional medical system for more than a hundred years. I was very glad that our persistent efforts finally persuaded the government. From this account of my own scientific background, I will begin this first lecture on Kampo medicine.

\section{Is Kampo CAM?}

Kampo was the backbone of Japanese medicine for more than 1500 years before it was excluded from the official medical education system after the Meiji Restoration of 1868. The new government decided to implement German medicine in order to thoroughly modernize the medical system of Japan.

The ordinary Japanese people, however, have never abandoned Kampo medicine and a certain degree of credit has been given to it throughout the history of modern Japan, as seen in the case of my uncle. Especially in the last 30 years of the 20th century, there has been a widespread resurgence of support in the population. The relatively recent introduction of the concept of CAM into the Western World has become the focus of hot debate. While medical practitioners of the West naturally put Kampo into the CAM category, many ordinary people in Japan would question calling Kampo either complementary or alternative.

The first reason for this is that, viewed from a historical Japanese perspective, Kampo is orthodox and Western medicine was imported as its alternative. Even though Western medicine is now no doubt the established mainstream in global medicine, if we take a culturally-relativistic position and pose a question as to what is orthodox and what is alternative medicine for a given culture, we can argue against assigning Kampo as alternative, at least from a historical point of view.

The second reason is related to the category of CAM in the West. The remedies and nutritional supplements that are thought canonical as CAM in the West often do not seem to have a solid systematic foundation in disease cognition or a healing philosophy. In other words, it seems to me that the Western concept of CAM is not defined in positive terms, but only in a negative way as, for example, being 'outside the mainstream' or a 'fringe' phenomenon. Kampo medicine, on the other hand, has its own system of disease cognition and healing philosophy. Since this system of cognition and healing philosophy is closer to the Japanese way of thinking, Kampo is, in a sense, mainstream or orthodox for us, at least conceptually. As such systems of cognition and philosophy, or paradigms, are sometimes non-commensurable with each other, it is not unreasonable to contend that Western medicine as a paradigm cannot call itself the global standard and categorize traditional Kampo medicine as its alternative. This is related to a larger epistemological subject, namely how those who are living in different paradigms understand each other's paradigm and categorize each other. Such a problem can be illuminated by an extreme example: can Buddhism and Shinto be regarded as alternatives to Christianity, which is globally orthodox?

Such big issues aside, I would like to point out from the start of this lecture a very specific point of difference between Kampo and modern Western medicine. It is the issue of 'reductionism' which has become a topic of interest these days. For example, it is well recognized that the power of modern Western drugs is based on chemistry. The great 
advancement of science and technology over the past 200 years has made it possible to chemically analyze, define and often synthesize the effective components that can cure diseases, the paradigmatic case being antibiotics. The miracle of this approach, however, has, unfortunately, nurtured an illusion that there should be at least one causal element that corresponds to one beneficial effect. This linear cause-effect picture of chemical remedies is now being challenged, even within the realms of Western science. In biological systems many of the cause-effect relationships would not be linear, but complex. The simplest of such complexities is that multiple-mutually interacting components exert a seemingly 'single effect'. I believe that many of the effects of Kampo medicines fall into this category. In the course of this series I would like to present evidence showing that the effects of certain Kampo remedies cannot be reduced to the effect of a single component but can only be understood as the result of the interaction of multiple components. This viewpoint is not at all supernatural or even holistic, and can just be seen as a more developed, or mature, form of (Western) rationalism. I believe one very good way to start to integrate Kampo and Western medicine can be found in this multiple-interacting components paradigm. I hope I am allowed to indulge in speculation that such a paradigm is more akin to the traditional Japanese pantheistic thinking than the Western Judeo-Christian monotheism.

On the other hand, however, I have no reservations in admitting that Kampo medicine has been complacent with being a local standard and has not sought evaluation by the methodology of the modern Western analytical paradigm. Even considering the situation within Japan, there is a great need to present the evidence of its utility in an analytical way. In other words, Kampo should not and cannot avoid meeting the challenge of the evidence-based medicine of the West. Philosophical problems aside, there is one major practical reason for this, that is related to the medical economy. As is well known, Japan is proud of its health-care system, based on compulsory national medical insurance coverage. From 1976 on, the system has approved 147 Kampo prescriptions for coverage by the national health insurance. This approval has met with much criticism, however, since these Kampo medicines had not passed clinical trials from phases I to III before being approved for commercial sale, a strict requirement for synthetic drugs. They were simply approved on the grounds that they had passed the test of a thousand years of historical experience. As the health insurance system of Japan is now in peril, a conflict of interest has arisen among those who lay claim to the limited financial resources. Such a circumstance has led to initiatives that require Kampo medicines to present the objective evidence of their utility and safety using the same methodology as that imposed on synthetic drugs.

This is, of course, a situational demand unique to Japan. But considering that Japan is a crossroads of Western and Eastern cultures, and because Kampo should become part of the intellectual legacy of humankind, there is no doubt that it is of global interest to test the utility and safety of Kampo and to universalize it by a unified standard of methodology.

Since such an approach exactly coincides with my own endeavors over many years, I feel that my efforts have now gained global support. There are, however, several important obstacles to overcome before such an objective evaluation can even be started. I would like to point out one of the biggest of such obstacles before I describe the details of Kampo medicine. Stated in a way that may astonish Western readers, with Kampo it is simply impossible to design a clinical trial which asks the question 'is such-and-such Kampo prescription effective or not for chronic hepatitis?' Out of ignorance, this approach has sometimes been taken in the West for the testing of Chinese herbal medicines, and one of the main themes of this lecture series is to persuade the readers that this kind of approach is not appropriate. As the readers may anticipate, the reason is the incommensurability of paradigms. The concept of chronic hepatitis simply does not exist in the Kampo paradigm. Thus, in designing a clinical trial for Kampo, it is desirable to take this point into account. Later in this lecture, I shall propose trial designs that take into consideration the paradigm within which Kampo prescriptions have their proper place. I shall also introduce to Western readers an outline of the evidence already obtained in Japan but not available in Western languages.

\section{The Historical Background of Kampo Medicine}

Kampo is traditional Japanese herbal medicine. It is based on traditional Chinese herbal medicine but developed into a unique form in Japan. I would like to note here that Kampo is not 'Japanese' medicine, nor is Chinese herbal medicine synonymous with 'Chinese' medicine. Especially noteworthy is the relationship between acupuncture and Kampo or herbal medicine. A different chapter should be devoted to this subject, but suffice it to point out here that these two modes have different origins and philosophies, and thus are not two aspects of integrated East Asian medicine. I think this non-integration derives from the socio-economic reason that the two different modes of therapies were practiced by two distinct professional guilds in pre-modern East Asian societies.

The official history states that the Chinese herbal medicine system was first imported into Japan from China via the Korean peninsula in $552 \mathrm{AD}$, but of course there must have been many more non-official social exchanges among the Chinese, Korean and Japanese peoples. After the official introduction, information on Chinese medicine continued to flow into Japan as part of various cultural exchanges between China and Japan. I think that Japanese Kampo was not very different from, and was in essence a copy of, contemporary Chinese herbal medicine until about 300 years ago.

In the midst of the Tokugawa era, around the early to middle 18th century, Japan experienced a so-called cultural renaissance, initiated by literature. Many scholars regard this as a sort of nationalistic movement in Japan, which 
has emerged intermittently throughout its history. In essence, such nationalism emerges to counter the non-critical acceptance or import of Chinese culture. For example, looking at the Confucian philosophy of that time, Japanese scholars tried to reject the then authoritative neo-Confucian $\mathrm{Zhu} \mathrm{Xi}$ interpretation and contended that the real meaning of the Confucian philosophy could only be understood by going back to the original real text. This 'text-critique' approach, championed by Ito, Jinsai and Ogiu, Sorai, was a Japanese style of positivism, which put into question the overtly speculative forms of the neo-Confucian philosophy of medieval China. Although this movement had elements that resonated with the contemporary Chinese re-interpretation of Confucianism, several literary scholars of Japanese classics, the most famous being Motoori, Norinaga, went so far as to reject any Chinese mind-set and call for a return to the "pure mind of ancient times' when Japan was supposedly free from Chinese influence.

These restoration efforts found echo in traditional medicine, where some Kampo leaders, among whom the most notable was Yoshimasu, Toudou, advocated a return to the original spirit of Chinese traditional medicine. It was emphasized by this school that Kampo prescriptions should avoid complexity in order to revive the fundamentals of simplicity, and also that a speculative or ideological approach should be avoided in preference to a positivistic one. Yoshimasu even declared that medicine was nothing more than 'effective prescriptions'. This would imply a purely pragmatic attitude that would accept any mode of therapy if it were effective, regardless of its philosophical background. Yamawaki, Toyo, another Kampo physician who was an ardent follower of Ogiu and an acquaintance of Yoshimasu, proved the inaccuracy of the traditional Chinese view of human anatomy (five solid and six tubal organs) by actual observations of the real human body of an executed prisoner. It is remarkable that such an undertaking had never been attempted in East Asia since ancient times. If reliance on actual observation and not on textual authority is to be taken as the hallmark of the modern scientific spirit, such an attitude in medicine began to burgeon in Japan around this period. Incidentally, it was just around the time of Yamawaki's bold attempt that Japanese physicians started to introduce Western medicine through the Dutch, who were the only Westerners officially permitted to trade with Japan. Thus, I think this turn in the thinking of Kampo medicine was, to some extent, an attempt on the part of Kampo practitioners in Japan to meet the challenge of a more positivistic Western medicine, which was an alternative medicine at that time. Especially astonishing to the Japanese in that period must have been the introduction of Western anatomy, which overtly contradicted the traditional Chinese view of the structure of the human body. Yamawaki's discovery had probably been motivated by a desire to know which view of the human body was true: the Chinese or the Dutch. Unlike China, where Western medicine was seen as barbaric CAM and gained only secluded and marginal acceptance, Japan in the Tokugawa era allowed Western medicine to be practiced alongside Chinese medicine. It was after this period that Kampo differentiated from traditional Chinese medicine and transformed itself into a uniquely Japanese medicine. In fact, the very term Kampo, which literally means 'Chinesestyle' in Japanese, was coined during this period to differentiate the traditional Japanese medicine from the then alternative Dutch-style (Rampo) medicine. Western readers should also note that the Chinese medicine now practiced in China is substantially different from the Kampo medicine practiced in Japan.

At this point, I would like to discuss in general terms how Kampo is different from Chinese medicine. I think one of the characteristics of 'Chinese' Chinese medicine is that it is deeply philosophical, or ideological. For any disease cognition and healing, philosophical accounts based on the Yin-Yang/Five Elements philosophy are required. Medical practice also proceeds more formally in China than in Japan. Another characteristic of 'Chinese' Chinese medicine is that, as it has evolved over such a long time and the tradition has been so well conserved, the cognition/healing system has become very complicated. Different approaches that have originated in different eras have simply piled up with little internal criticism. In contrast, Japanese Kampo is more practical and informal, relying little on Taoist or other Chinese philosophy. Western readers should thus be cautioned against the idea that any Chinese herbal medicine is based on the Chinese paradigm of cognition and practice. The Japanese paradigm of Kampo is little dependent on, if not free from, the Yin-Yang/Five Elements paradigm. I think that Kampo can be characterized as a simplified, positivistic and pragmatic version of Chinese herbal medicine.

After the Meiji Restoration, Japan decided to take on a definitive route to modernization, or Westernization. As part of its thorough efforts to dispel many traditions as pre-modern, the Meiji government decided to implement the German system in official medical education and practice, and at the same time exclude the Kampo system as pre-modern. This move should not be regarded as either ideological or political, however. By this time, it had long been recognized that Western medicine had distinct superiority over traditional Japanese medicine, including Kampo, most notably shown in vaccinations and military surgery. Thus, the decision taken by the government to transform the medical system was basically pragmatic. I suspect that it was mainly from the viewpoints of military and public health-where Kampo was clearly inferior - that the Meiji leaders turned to German medicine. Here, I would like to recount one anecdote that shows how open-minded those Meiji leaders were. In 1878, the government asked representatives of the Western and Kampo schools to join into an open competition to heal beriberi, which was a prevalent disease at that time with an unknown etiology. The two schools were asked to first treat a similar number of patients separately. The patients who did not improve were then given over to treatment by the other school. Although the methodology was remarkably fair and scientific, the results seemed equivocal, and understandably 
so, since neither school had any idea at that time that the disease was mainly caused by a low dietary intake of thiamine, subsequently discovered by Suzuki, Umetaro in 1910. Thus, Kampo medicine could not gain acceptance in competition with Western medicine in the new age, and in 1883 a law was even decreed that no Kampo physician was officially licensed as a medical doctor. Nevertheless, Japanese people never abandoned Kampo herbs, and Kampo medicine was continuously supported as a part of Japanese culture, just as Kabuki and Noh have never been replaced by Western theater. On the other hand, many academic departments of Japanese universities undertook modern scientific analysis of traditional herbs, even though Kampo was not regarded as official medicine. One remarkable achievement was the extraction, structural determination and then synthesis of ephedrine, the major chemical constituent of Ephedrae Herba by Nagai, Nagayoshi around 1900. It is interesting to note here that since Kampo was regarded not as a purely Japanese tradition, there was no resurgence of Kampo at the time of ultranationalism based on Shinto which led to the disastrous war between Japan and China and the US. People preserved Kampo as part of Japanese culture not because it was a sacred tradition to be faithfully observed but simply because some of the Kampo remedies were quite effective.

Even so, it took about 100 years for Kampo medicine to make a comeback and receive official attention around 1970. As mentioned above, this coincided with my graduation from medical school, and acquiring 'bilingual' ability. This move was influenced by several changes in Japanese society. First, the disease structure of the Japanese population changed drastically around these years. No infectious diseases remained as major killers and the important problems associated with the rapidly aging population of Japan were cancer and cardiovascular disease, like any developed Western country. Second, as environmental contamination became a global problem around this period, many of the adverse effects of synthetic Western drugs were perceived as contamination by chemicals. The case of thalidomide is representative of this. The drug, which was developed in Germany and then imported into Japan mainly as a sedative, was later found to be teratogenic in Germany. It was recalled immediately in Germany and was even disavowed by the FDA in the USA. However, due to bureaucratic misconduct and negligence on the part of pharmaceutical companies, it remained on the market in Japan for several years after the adverse effects had been reported. The legacy of many terrible photographs of affected babies from this incident taught the Japanese public the gravity of the adverse effects of 'magic' drugs created by chemical synthesis. The age-proven herbal remedies of Kampo newly regained the attention of the public at last. A third and intriguing reason may be that at around this period Japan had begun to feel that there was nothing more to learn from the West. The 'last frontier' became its own tradition, which would have been in peril of extinction if the lack of support and interest had remained as it was.
It was Dr Taro Takemi, then President of the Japanese Association of Physicians, who pioneered this epic change. He advocated the utility of Kampo medicine and succeeded in persuading the government to approve 147 kinds of Kampo herbal extracts for official coverage by the national health care insurance system in 1976.

Several specialists criticized this initiative by Dr Takemi as being outside the law. While any other 'Western' drugs prescribed by doctors could be approved only after three phases of clinical trials, Kampo herbal extracts had not been required to undergo such a process. I, however, disagree with this criticism, based on the history from the Meiji period (post 1867) of the administrative control of medical drugs. Although the Meiji government excluded Kampo medicine from official medical education, it did not by any means ban Kampo medicine. On the contrary, various herbal medicines have never ceased to be prescribed by Kampo practitioners without interference from the authorities. In fact, increasing numbers of Kampo herbal medicines have long been registered in Japanese Pharmacopoeia, the official registry of all medicines usable in Japan. When the National Health Insurance System was implemented in Japan in 1960 to establish universal compulsory coverage for anyone with Japanese nationality, the government permitted any herbal medicines registered in Japanese Pharmacopoeia to be combined, becoming 'brewed' medicines. In other words, the Japanese government has long recognized Kampo as a tradition, with Kampo herbs categorized as drugs separate from Western synthetic drugs. I find this a remarkable policy for the Japanese government to implement. In this context, the criticism of the introduction of Kampo herbs into the Japanese medical system as extra-legal cannot be justified, since Kampo herbal brews have never been illegal in Japan and the newly approved herbal extracts were nothing more than herbal brews manufactured in the drug manufacturing plants. In this respect, I would also like Western readers to note that all the Kampo herbs now covered by the National Health Insurance System of Japan are extracts manufactured under strict quality control.

This justification based on cultural tradition, of course, cannot be universalized, as globally no one can justify the efficacy of any drug simply because it has been used for a long period in some geographic region. The clinical trial procedure now established as a global standard is a very robust, if not invincible, principle. This does not mean, however, that those traditional remedies, which have not undergone such formal trials, are ineffective. On the contrary, I think that we can be proud of the wisdom that led to the preservation of Kampo herbs, a wisdom that was cultivated by the history and culture of Japan. Moreover, it is thanks to this wisdom that a wide range of Japanese people now benefit from Kampo herbs. After their official recognition as medical drugs, chemical and pharmacological research on Kampo herbs has made dramatic progress in Japan. The chemical components of each herb are now analyzed in such detail that I think we can now safely talk about the new science of 
Kampo as the science of a complex, or compound, drug. It is with this background that the government decided to introduce Kampo into the core curriculum of medical and pharmaceutical education in 2002. Now it is expected that all medical and pharmaceutical students in Japan will acquire an understanding of the basics of Kampo medicines. Looking back through history, I think that the time has come for Kampo medicine to be approached from a fresh global perspective. Kampo medicine integrated into Western medicine may not be Kampo any more, but at the same time Western medicine will never be the same.

\section{Background Reading}

It is truly regrettable that there is virtually no authoritative reference in English of Kampo medicine. Even for Chinese herbal medicine, I am not aware of any references in English I can recommend without reservation. There is an English translation of my book (1), part of which is outdated, but my thinking is still basically the same. Many books are available in Japanese on Kampo (see, for example, ref. 2). Also available in Japanese are recent reviews of mine $(3,4)$.

\section{General References}

1. Terasawa K. Kampo: Japanese-Oriental Medicine-Insights from Clinical Cases (co-translated by Helmut Bacowsky and Arendt Gerz), Tokyo, Japan: Standard McIntyre KK 1993.

2. Terasawa K, Kita T. EBM Kampo. Tokyo, Japan: Ishiyaku-Shuppan KK.

3. Terasawa K. Construction of medicine for the new century: Integration of Western and Eastern Medicines, Nihon Toyo Igaku Zasshi. Jpn J Oriental Med 2002;53:11-35.

4. Terasawa K. Another medicine: Kampo Igakuno-Ayumi. Prog Med 2000;192:309-12. 


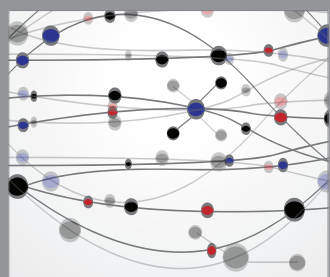

The Scientific World Journal
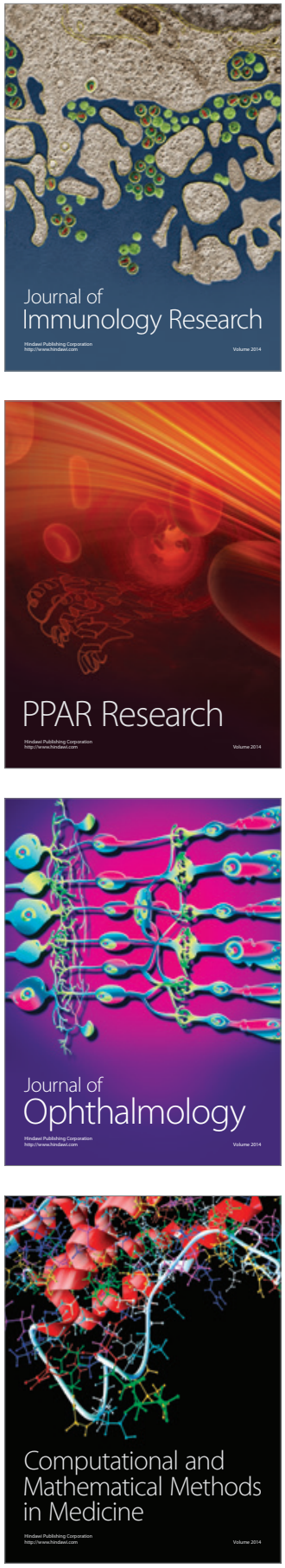

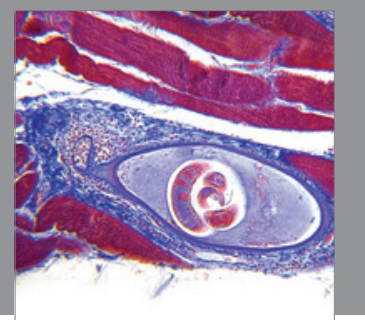

Gastroenterology

Research and Practice
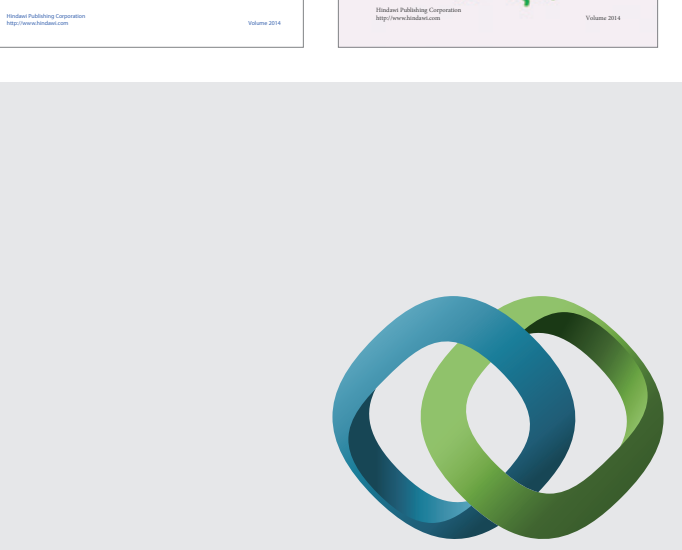

\section{Hindawi}

Submit your manuscripts at

http://www.hindawi.com
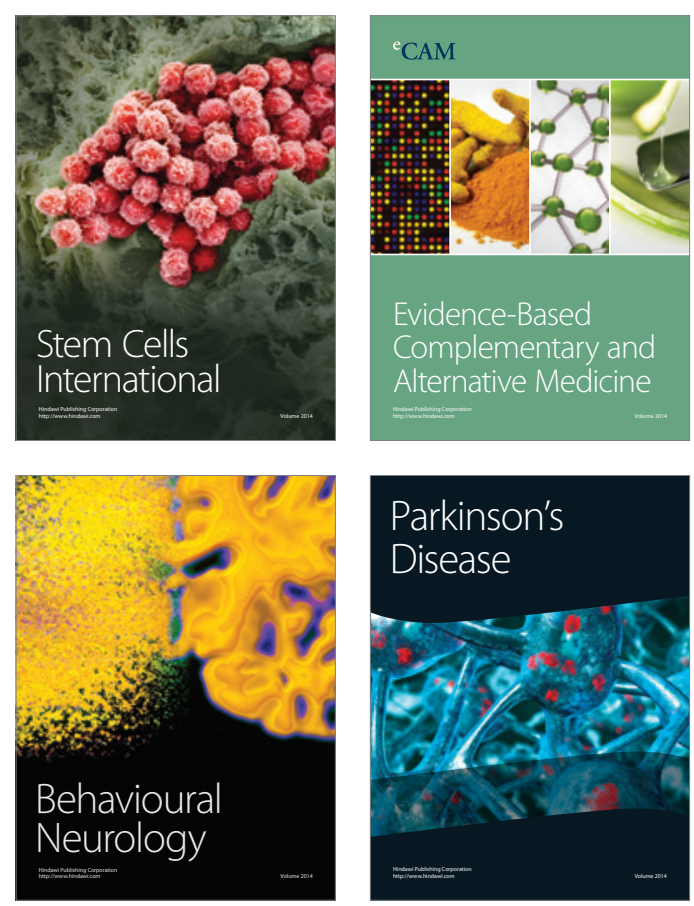

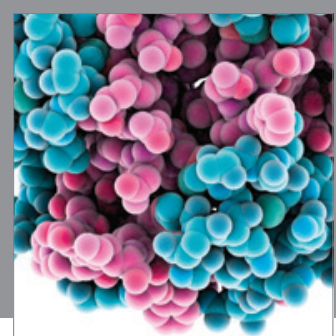

Journal of
Diabetes Research

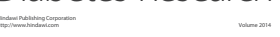

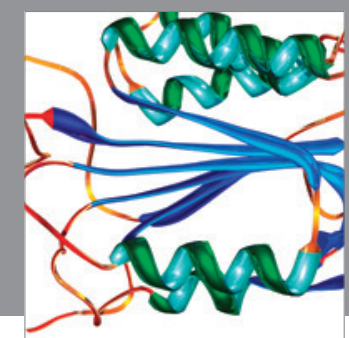

Disease Markers
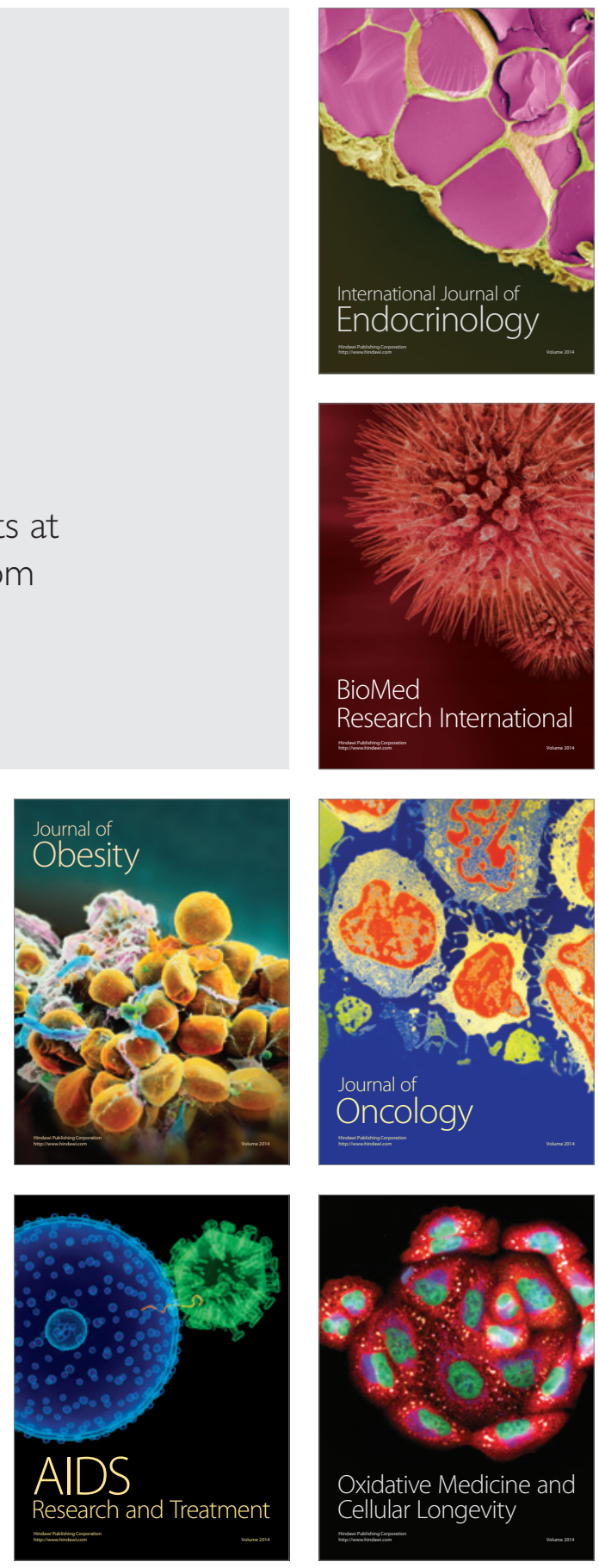